\title{
Energy Dissipation to Tungsten Surfaces upon Hot-Atom and Eley-Rideal Recombination of $\mathbf{H}_{2}$
}

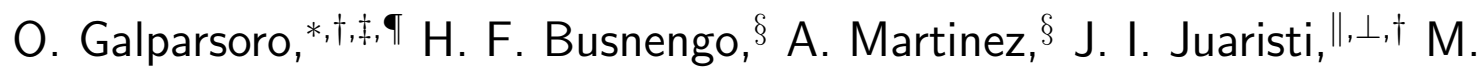 \\ Alducin, ${ }^{\perp, \dagger}$ and P. Larrégaray \\ Donostia International Physics Center (DIPC), Paseo Manuel de Lardizabal 4, 20018 \\ Donostia-San Sebastián, Spain, Université de Bordeaux, ISM, UMR 5255, F-33400 \\ Talence, France, CNRS, ISM, UMR5255, F-33400 Talence, France, Instituto de Física \\ Rosario (IFIR) CONICET-UNR, Esmeralda y Ocampo, 2000 Rosario, Argentina, \\ Departamento de Física de Materiales, Facultad de Químicas (UPV/EHU), Apartado 1072, \\ 20080 Donostia-San Sebastián, Spain, and Centro de Física de Materiales CFM/MPC \\ (CSIC-UPV/EHU), Paseo Manuel de Lardizabal 5, 20018 Donostia-San Sebastián, Spain \\ E-mail: o.galparsoro@gmail.com
}

\footnotetext{
*To whom correspondence should be addressed

$\dagger$ Donostia International Physics Center (DIPC), Paseo Manuel de Lardizabal 4, 20018 Donostia-San Sebastián, Spain

$\ddagger$ Université de Bordeaux, ISM, UMR 5255, F-33400 Talence, France

ฯ CNRS, ISM, UMR5255, F-33400 Talence, France

§Instituto de Física Rosario (IFIR) CONICET-UNR, Esmeralda y Ocampo, 2000 Rosario, Argentina

"Departamento de Física de Materiales, Facultad de Químicas (UPV/EHU), Apartado 1072, 20080 Donostia-San Sebastián, Spain

${ }^{\perp}$ Centro de Física de Materiales CFM/MPC (CSIC-UPV/EHU), Paseo Manuel de Lardizabal 5, 20018 Donostia-San Sebastián, Spain
} 


\title{
1 Introduction
}

\begin{abstract}
Adiabatic and nonadiabatic quasi-classical molecular dynamics simulations are performed to investigate the role of electron-hole pair excitations in Hot-Atom and EleyRideal $\mathrm{H}_{2}$ recombination mechanisms on $\mathrm{H}$-covered $\mathrm{W}(100)$. The influence of the surface structure is analyzed by comparing with previous results for $\mathrm{W}(110)$. In the two surfaces, Hot-atom abstraction cross sections are drastically reduced due to the efficient energy exchange with electronic excitations at low incident energies and low coverage, while the effect on Eley-Rideal reactivity is negligible. As the coverage increases, the projectile energy is more efficiently dissipated into the other adsorbates. Consequently, the effect of electronic excitations is reduced. As a result, the reactivity and final energy distributions of the formed $\mathrm{H}_{2}$ molecules are similar for both abstraction mechanisms.
\end{abstract}

\section{Keywords}

Gas-surface interactions, Eley-Rideal reactions, scattering, potential energy surfaces, molecular dynamics, electron-hole pair excitations, phonons, energy dissipation

In the last decades the description of surface chemical processes has undergone such a development that theoretical methods are achieving the required accuracy to become a powerful tool for industrial engineering. ${ }^{1}$ Nevertheless, many aspects within the theoretical description and understanding of gas-surface interactions still need to be improved. In particular the dynamics of such interactions might play a crucial role in the final surface reactivity. ${ }^{2,3}$ In this context, the validity of the commonly employed Born-Oppenheimer approximation (BOA) is an important issue, which is still under scrutiny. Although satisfactory agreement between electronically adiabatic simulations and experiments has been achieved in some cases, ${ }^{4-9}$ there is experimental evidence of relevant amount of electron-hole $(\mathrm{e}-\mathrm{h})$ pair excitations being created when chemical compounds interact with metal surfaces. ${ }^{10,11}$ 
This raises the question of to what extend electronically nonadiabatic interactions influence gas-surface processes. Their influence might depend on the particular process under study. ${ }^{12}$ For instance, in fast processes such as dissociative adsorption or scattering, electronic excitations are in general found to negligibly affect the probabilities of the processes. ${ }^{13-19}$ In contrast, nonadiabaticity might be crucial in order to describe final energy distributions of light scattered species ${ }^{20-23}$ as well as processes involving hyperthermal diffusion of the gas compound on the surface. ${ }^{24-27}$ The characteristics of the system of interest such as the chemical species involved, ${ }^{25}$ the surface coverage, ${ }^{26}$ and the surface temperature ${ }^{28}$ might also be relevant regarding the effect of electronic excitations.

A process for which experiments have suggested influence of electronic excitations is the hydrogen abstraction process, ${ }^{29}$ in which an adsorbed $\mathrm{H}$ is extracted from the surface by reaction with a gas $\mathrm{H}$ atom. It may proceed through Eley-Rideal (ER) or Hot-Atom (HA) mechanisms. The former refers to a single collision reactive event, and therefore it is characterized by ultrafast reaction times. Although this characteristic suggests low influence of e-h pair excitations, theoretical calculations for ER recombination on W(100) and W(110) showed that due to the high exothermicity, a large amount of energy is released which substantially excites electrons. ${ }^{22}$ While small influence on the reactivity was predicted, the final energy of the formed molecules was affected. HA recombination, on the other hand, is governed by hyperthermal diffusion of the $\mathrm{H}$ atom onto the surface prior to abstraction. Comparisons between experiments and kinetics models ${ }^{30-33}$ have suggested abstraction to essentially proceed via the HA process for various metals. Moreover, adiabatic molecular dynamics simulation ${ }^{33-36}$ studies predicted higher contribution of HA recombination comparing to ER for low incidence energies of the gas atom and low coverages. However, all these works neglected e- $\mathrm{h}$ pair excitations during the recombinative process. Recently, nonadiabaticity was introduced in $\mathrm{H}$ recombination on W(110) and the results showed that both ER and HA mechanisms contribute similarly to the total abstraction cross section regardless the incidence energy and coverage. ${ }^{26,27}$ 
Different $a b$ initio theories have been developed to deal with $\mathrm{e}-\mathrm{h}$ pair excitations in molecular processes on metal surfaces. ${ }^{37-43}$ Among them, the local density friction approximation (LDFA) ${ }^{12,13,44}$ offers a good compromise between accuracy of results and simplicity of implementation. This approach has been used to study the energy dissipation in different processes on metal surfaces such as adsorption, ${ }^{24,45,46}$ scattering, ${ }^{19,23,47,48}$ dissociation, ${ }^{13-17}$ recombination, ${ }^{22,26,27,49,50}$ and femtosecond laser induced desorption. ${ }^{51-54}$

Making use of this methodology, we investigate here nonadiabatic effects on both abstraction mechanisms for $\mathrm{H}_{2}$ recombination on $\mathrm{H}$-covered $\mathrm{W}(100)$ and $\mathrm{W}(110)$ surfaces. The influence of surface geometry and surface coverage is analyzed. In order to do so, a density functional theory (DFT) based multiadsorbate potential energy surface (PES) for $\mathrm{H}$ interacting with H-covered W(100) has been constructed following the Corrugation Reducing Procedure $(\mathrm{CRP})^{55-57}$ method. The article is structured as follows. Methodology and details for the construction of the PES and dynamics simulation calculations are described in Sections 2.1 and 2.2, respectively. In Sections 3.1 and 3.2, results of the dynamics calculations are analyzed. Finally, Section 4 concludes.

\section{Theoretical methods}

\subsection{Potential energy surface for $\mathrm{H}+\mathrm{H} / \mathrm{W}(100)$}

The PES constructed to study $\mathrm{H}_{2}$ dissociation on W(100) in Ref. 58 has been extended in order to simulate $\mathrm{H}_{2}$ recombination on $\mathrm{H}$-covered $\mathrm{W}(100)$. Let us refer to the former as the $\mathrm{H}_{2} / \mathrm{W}(100)$ PES and to the latter as the $\mathrm{H}+\mathrm{H} / \mathrm{W}(100)$ PES. For dissociative adsorption,

internuclear distances $r$ in the range $\left[r_{e q} / 2,2 r_{e q}\right]$ were considered, where $r_{e q}=0.75 \AA$ is the equilibrium internuclear distance of $\mathrm{H}_{2}$. However, in the case of $\mathrm{HA}$ and ER recombination processes, the upper limit in the previous $r$-grid has to be increased in order to incorporate atomic configurations at the entrance channel (one atom far from the surface) in the interpolation scheme. To compute the extra $\mathrm{H}$ - and $\mathrm{H}_{2}$-surface potential energies, DFT cal- 
culations were carried out with the Vienna Ab Initio Simulation Package (VASP), ${ }^{59-63}$ using the same $2 \times 2$ supercell and parameters as in the construction of the $\mathrm{H}_{2} / \mathrm{W}(100)$ PES. ${ }^{58}$ The PW91 ${ }^{64,65}$ functional was used to describe electronic exchange and correlation. Electron-ion interactions were described through ultrasoft pseudopotentials. ${ }^{66}$ Since the $\mathrm{H}_{2}$ abstraction processes occur on a non-magnetic metal surface as W, spin-polarized DFT calculations are only required whenever the interatomic distance is $r>1.6 \AA$ and at least one of the atoms is relatively far from the surface, i.e., at heights $Z>2.6 \AA$.

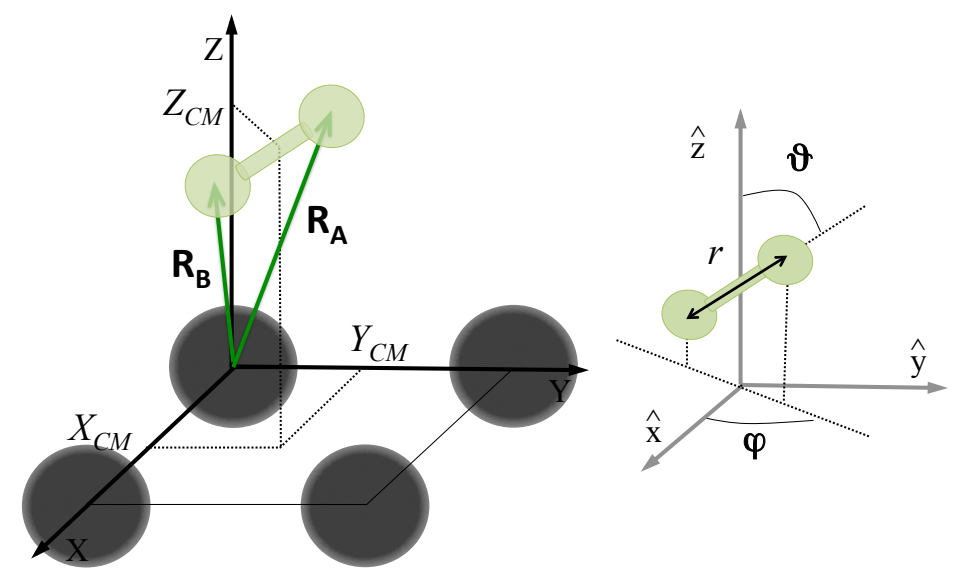

Figure 1: The coordinate system used for the $\mathrm{H}_{2} / \mathrm{W}(100)$ and $\mathrm{H}+\mathrm{H} / \mathrm{W}(100) \mathrm{PESs}$. $\mathrm{H}$ and $\mathrm{W}$ atoms are represented as green and gray spheres, respectively. The origin of the coordinate system is placed on a $\mathrm{W}$ surface atom.

As done for the construction of the $\mathrm{H}_{2} / \mathrm{W}(100) \mathrm{PES},{ }^{58}$ the $\mathrm{H}+\mathrm{H} / \mathrm{W}(100)$ PES was obtained by interpolating two-dimensional (2D) DFT energy grids in the molecular center of mass $(\mathrm{CM})$ height $Z_{C M}$ and internuclear distance $r$ that were calculated for the following 28 different molecular configurations:

- Three configurations over top site $\left(X_{C M}=0, Y_{C M}=0\right): \vartheta=0^{\circ} ; \vartheta=90^{\circ}$ with $\varphi=0^{\circ}$ and $\varphi=45^{\circ}$.

- Three configurations over hollow site $\left(X_{C M}=a / 2, Y_{C M}=a / 2\right): \vartheta=0^{\circ} ; \vartheta=90^{\circ}$ with $\varphi=0^{\circ}$ and $\varphi=45^{\circ}$. 
- Three configurations over bridge site $\left(X_{C M}=a / 2, Y_{C M}=0\right): \vartheta=0^{\circ} ; \vartheta=90^{\circ}$ with $\varphi=0^{\circ}$ and $\varphi=90^{\circ}$.

- Seven configurations over top to hollow site $\left(X_{C M}=a / 4, Y_{C M}=a / 4\right): \vartheta=0^{\circ} ; \vartheta=45^{\circ}$ with $\varphi=45^{\circ}, \varphi=90^{\circ}, \varphi=135^{\circ}$ and $\varphi=225^{\circ}$; and $\vartheta=90^{\circ}$ with $\varphi=45^{\circ}$ and $\varphi=135^{\circ}$.

- Seven configurations over bridge to hollow site $\left(X_{C M}=a / 2, Y_{C M}=a / 4\right): \vartheta=0^{\circ} ; \vartheta=45^{\circ}$ with $\varphi=0^{\circ}, \varphi=90^{\circ}$, and $\varphi=270^{\circ}$; and $\vartheta=90^{\circ}$ with $\varphi=0^{\circ}, \varphi=45^{\circ}$, and $\varphi=90^{\circ}$.

- Five configurations over top to bridge site $\left(X_{C M}=a / 4, Y_{C M}=0\right)$ : $\vartheta=0^{\circ} ; \vartheta=45^{\circ}$ with $\varphi=0^{\circ}$ and $\varphi=180^{\circ}$; and $\vartheta=90^{\circ}$ with $\varphi=0^{\circ}$ and $\varphi=90^{\circ}$.

In the above list, a molecular configuration is defined by the molecular orientation $(\vartheta, \varphi)$ and the position of the molecular CM over the surface unit cell $\left(X_{C M}, Y_{C M}\right)$ (see Fig. 1 for a sketch on the coordinate system). The $2 \mathrm{D}-\left(Z_{C M}, r\right)$ grids were here extended to $r_{\max }=3.0 \AA$, which is the value at which the interpolation function $I^{6 D}$ (see below) is found to vanish. Next, the PES interpolation was performed by means of the corrugation-reducing procedure (CRP) method, ${ }^{55-57}$ in which the potential $V^{6 D}$ is described as the sum of the atom-surface interaction $V^{3 D}$ and the interpolation function $I^{6 D}$ that represents the effective $\mathrm{H}-\mathrm{H}$ interaction potential on the rigid surface,

$$
V^{6 D}\left(\mathbf{R}_{i}, \mathbf{R}_{j}\right)=\sum_{i} V_{i}^{3 D}\left(\mathbf{R}_{i}\right)+I^{6 D}\left(\mathbf{R}_{i}, \mathbf{R}_{j}\right)
$$

where $\mathbf{R}_{i}$ is the position vector of atom $i$. By this method most of the corrugation of a gassurface PES is reduced before doing the interpolation, and as a consequence, the accuracy of the interpolation is improved.

The $V^{3 D}$ atom-surface DFT energies were computed for the same heights $Z$ and surface sites, using spin polarized DFT for $Z>2.6 \AA$. Moreover, the PES was extended to describe possible subsurface penetration of $\mathrm{H}$ down to $-4 \AA(Z=0$ is defined by the height of the topmost surface layer). For negative values of $Z_{C M}$, the interpolation function $I^{6 D}$ was assumed 
to be constant and equal to the value of $I^{6 D}$ at $Z_{C M}=0 \AA$. Therefore, the $\mathrm{H}+\mathrm{H} / \mathrm{W}(100)$ PES does not accurately describe the $\mathrm{H}-\mathrm{H}$ interaction within the bulk. However, our goal was to model recombination of atoms that penetrate into the bulk and later return to the surface where they recombine. In fact, in the quasi-classical molecular dynamics simulations (QCT) we did not find any event in which two atoms approach to each other inside the metal, due to the high repulsion they experience when they are embedded in a high electronic density environment.

Once the DFT energy data were calculated, $I^{6 D}$ was obtained by subtracting the atomsurface interactions $V^{3 D}$ from the molecule-surface potential $V^{6 D}$. Then, the continuous representation of $I^{6 D}$ on $\left(X_{C M}, Y_{C M}, Z_{C M}, r, \vartheta, \varphi\right)$ is obtained by interpolating $I^{6 D}$ over $Z_{C M}$ and $r$ for each of the above listed molecular configurations using 2D-cubic splines, as a first step. Next, symmetry-adapted expansions of trigonometric functions are employed for the interpolation over $\vartheta$ and $\varphi$ on each surface site. ${ }^{55,57}$ Finally, the interpolation over $X_{C M}$ and $Y_{C M}$ is done with 2D-periodic cubic splines. The atom-surface energies were interpolated by using a 3D-cubic spline.

The resultant $\mathrm{H}+\mathrm{H} / \mathrm{W}(100)$ PES reproduces properly the most stable atomic adsorption site located on the bridge position with a height $Z=1.107 \AA,{ }^{67-72}$ as well as the DFT chemisorption energy of $3.09 \mathrm{eV}$. The molecular hydrogen adsorption energy of $-1.63 \mathrm{eV}$ at $\Theta=0.5 \mathrm{ML}$ compares relatively well with the recent value of $-1.78 \mathrm{eV}$ calculated by $\mathrm{Pi}-$ azza et al. for the same coverage, but for the reconstructed W(100) surface. The zero point energy (ZPE) components were calculated following the $\mathrm{X}, \mathrm{Y}$, and $\mathrm{Z}$ mode decomposition method ${ }^{73,74}$ for one isolated adsorbate. The ZPEs of the modes associated to parallel movement along the $\mathrm{X}$ and $\mathrm{Y}$ directions are 69 and $40 \mathrm{meV}$, respectively. The ZPE for vibrational motion normal to the surface is $67 \mathrm{meV}$. These values are in good agreement with theoretical ${ }^{75,76}$ and experimental ${ }^{68,70,77,78}$ values reported previously for $\mathrm{H} / \mathrm{W}(100)$.

In order to evaluate the quality of the constructed $\mathrm{H}+\mathrm{H} / \mathrm{W}(100)$ CRP PES, additional DFT energy calculations were performed and compared with the CRP values. Since the ER 


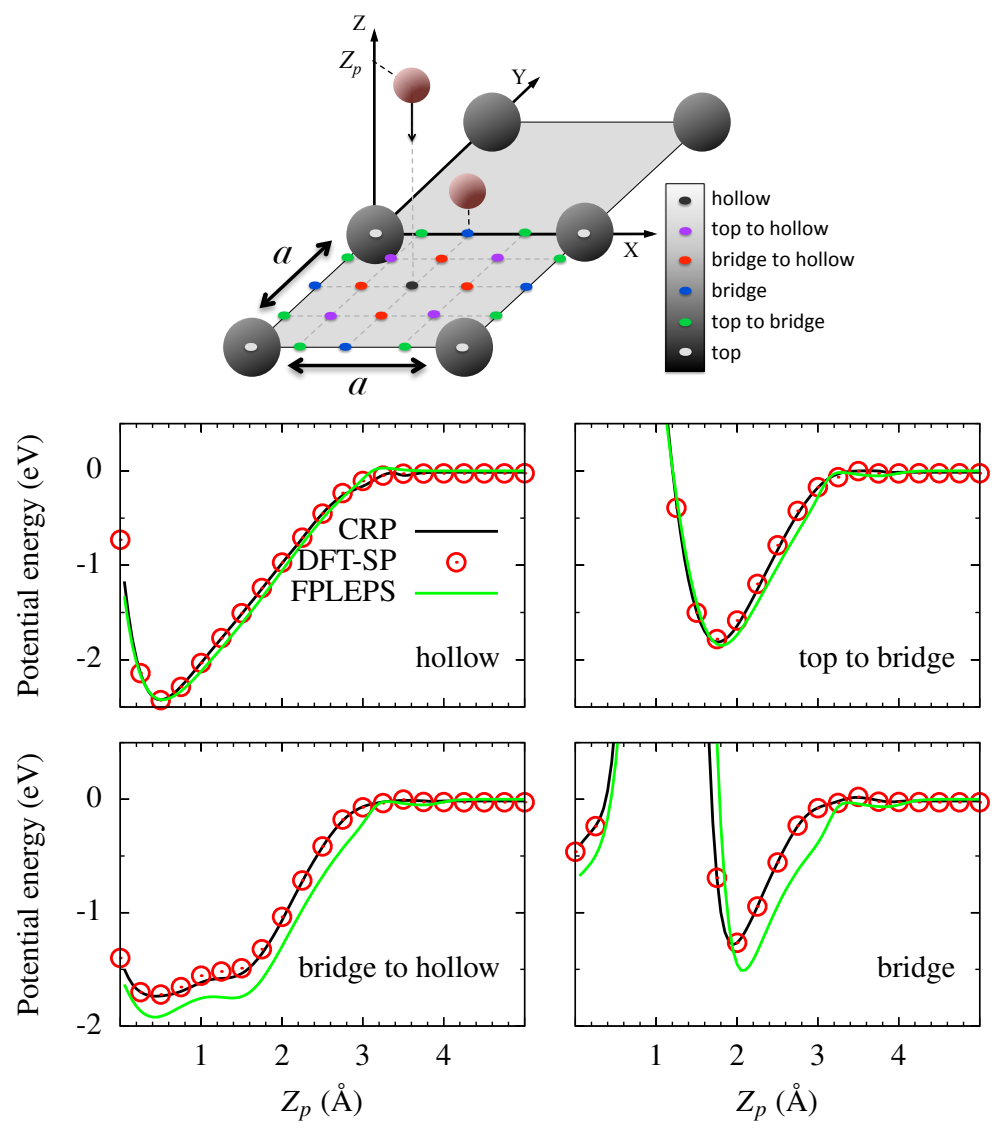

Figure 2: Comparison between the CRP (black curves), the FPLEPS (green curves), and the spin-polarized DFT calculations (red circles) in the ER entrance channel for different $\left(X_{p}, Y_{p}\right)$ positions of the projectile: top to bridge $(0, a / 4)$, bridge $(a / 2,0)$, hollow $(a / 2, a / 2)$ and bridge to hollow $(a / 2, a / 4)$, where $a=3.17 \AA$ is the lattice constant. The different $\left(X_{p}, Y_{p}\right)$ positions and the coordinate system defining $Z_{p}$ are indicated in the top figure. 

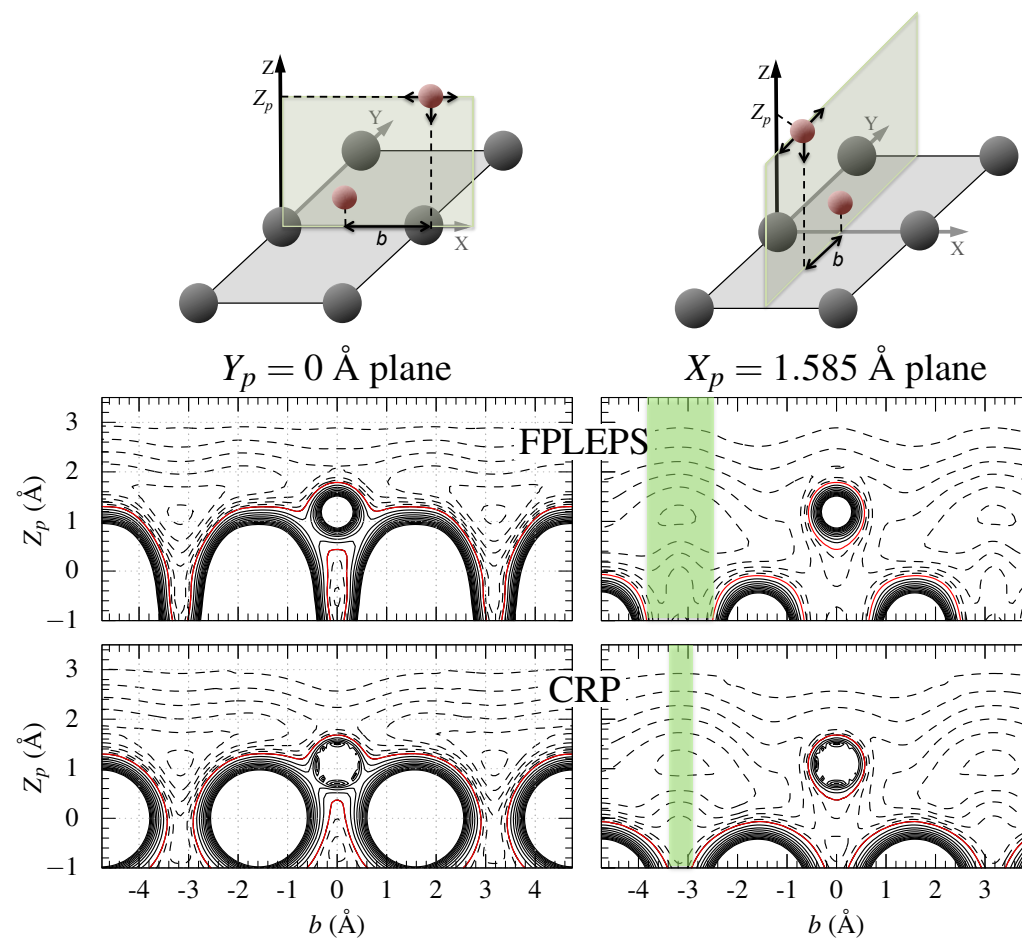

$X_{p}=1.585 \AA$ A plane

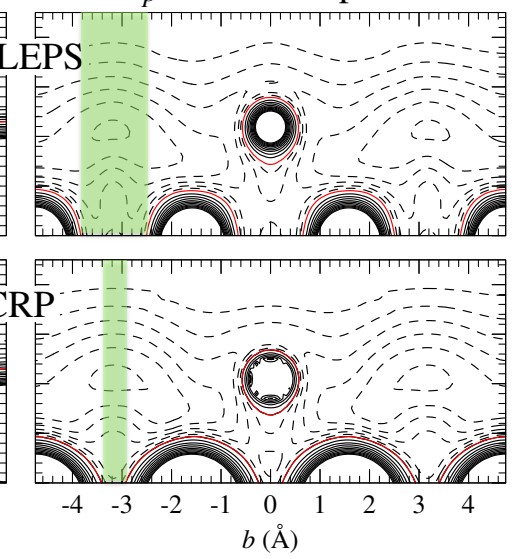

Figure 3: 2D $\left(b, Z_{p}\right)$-cuts of the FPLEPS (top) and the CRP (bottom) H+H/W(100) PESs. The adsorbate sits on its equilibrium position and the projectile spans the $\left(b, Z_{p}\right)$ plane for $X_{p}=1.585 \AA$ (right panels) and $Y_{p}=0$ (left panels). Full lines (dashed lines) are positive (negative) isovalues separated by $1 \mathrm{eV}(0.5 \mathrm{eV})$. The zero potential energy is depicted by a red line. Top right (left) scheme indicates the $\left(b, Z_{p}\right)$ plane for $X_{p}=1.585 \AA\left(Y_{p}=0\right)$ and the coordinate system used. The target is located at bridge. 
dynamics was previously studied by using a different $\mathrm{H}+\mathrm{H} / \mathrm{W}(100) \mathrm{PES},{ }^{58,74}$ we also found meaningful to compare and evaluate the differences between our CRP PES and the one used in these references, which is based in the flexible periodic London-Eyring-Polanyi-Sato (FPLEPS) method. ${ }^{79-81}$ In doing such a comparison, note that both PESs were constructed using DFT data calculated with the same parameters. Fig. 2 shows 1D-cuts of the PESs as a function of the projectile height $Z_{p}$ for a given $\left(X_{p}, Y_{p}\right)$ position while the target is fixed at the bridge site. The agreement between the DFT data and the CRP PES is very good (discrepancies being lower than $60 \mathrm{meV}$ ), while the FPLEPS is less accurate (discrepancies being lower than $300 \mathrm{meV}$ ). The corresponding root mean square deviations (RMSD) for the 1D cuts of the FPLEPS and CRP PESs shown in Fig. 2 are 195 and $22 \mathrm{meV}$, respectively. In total, we evaluated 192 spin-polarized DFT energy data that correspond to configurations in which the target was located at bridge, bridge to hollow, and top to bridge positions, while the projectile approached the surface from $3.5 \AA$ down to $0 \AA$ over top, bridge, hollow, top to bridge, bridge to hollow, and top to hollow positions. The calculated DFT energies were then compared with the values predicted by the FPLEPS and CRP PESs, but considering only the energies relevant for the present work $(<5.0 \mathrm{eV})$. The corresponding RMSD are 300 meV for the FPLEPS and $50 \mathrm{meV}$ for the CRP. Similar RMSD values were obtained for the $\mathrm{H}+\mathrm{H} / \mathrm{W}(110)$ FPLEPS $(230 \mathrm{meV})$ and CRP (55 meV) PESs. ${ }^{73}$

Fig. 3 shows 2D- $\left(b, Z_{p}\right)$ cuts of the FPLEPS (top) and CRP (bottom) PESs, along the $Y_{p}=0 \AA$ and $X_{p}=1.585 \AA$ planes that provide useful information on the properties of both $\mathrm{H}+\mathrm{H} / \mathrm{W}(100)$ PESs. The parameter $b$ is defined as the impact parameter (see top schemes in Fig. 3). The adsorbate sits on its equilibrium position, whereas the position of the projectile varies within the $Y_{p}=0 \AA$ and $X_{p}=1.585 \AA\left(b, Z_{p}\right)$-planes depicted in Fig. 3. The two PESs are qualitatively very similar. For instance,both PESs are attractive in the region around the unoccupied bridge sites and are less attractive in the vicinity of the target. Also, the repulsive part of both PESs is closely related with the position of the $\mathrm{W}$ nuclei and the target atom. Still, some discrepancies are observed. On the one hand, close to 
the target position, the topology of the two PESs differs due to the lower adsorption height of the target in the CRP PES $\left(Z_{t}=1.107 \AA\right.$ vs. $Z_{t}=1.2 \AA$ in the FPLEPS). On the other hand, the repulsive part around the second layer W atoms is larger in the CRP PES than in FPLEPS (see the reduction of the attractive green areas in the right panels of Fig. 3).

In order to account for finite coverages, the (rigid-surface) multiadsorbate potential $V\left(\left\{\mathbf{R}_{i}\right\}\right)$ is approximated by the following two-H terms expansion, ${ }^{26,35,36,82}$

$$
V\left(\left\{\mathbf{R}_{i}\right\}\right)=\sum_{i=1}^{N} V^{3 D}\left(\mathbf{R}_{i}\right)+\sum_{i=1}^{N} \sum_{j>i}^{N} I^{6 D}\left(\mathbf{R}_{i}, \mathbf{R}_{j}\right)
$$

where $N$ is the total number of $\mathrm{H}$ atoms (i.e., the projectile plus the adsorbates). Therefore, three-H interactions are disregarded. This approximation limits the validity of the multiadsorbate PES to low coverage conditions, although higher coverages up to $2 \mathrm{ML}$ are thermodynamically favourable ${ }^{83}$ and therefore can be expected to be present at equilibrium conditions. In this work, we study the 0.5 and $1.0 \mathrm{ML}$ coverages depicted in Fig. 4. The same adsorption positions as well as ZPEs as in the zero coverage limit are found for both coverages. The reason is that the adsorbates do not interact at internuclear distances larger than $3.0 \AA$, in agreement with DFT calculations.

Fig. 4 shows $2 \mathrm{D}\left(b, Z_{p}\right)$-cuts along the $X_{p}=1.585 \AA, Y_{p}=0$ and $Y_{p}=X_{p}-1.585 \AA$ planes of the $\mathrm{H}+\mathrm{H} / \mathrm{W}(100) \mathrm{PES}$ at $\Theta=0.5$ and $1.0 \mathrm{ML}$ coverages. The comparison with Fig. 3 shows that at $\Theta=0.5$ and 1.0 ML coverages, the interaction potential close to the target is very weakly affected by the coverage. In the $X_{p}=1.585 \AA$ and $Y_{p}=0 \AA$ planes, at 0.5 (1.0) ML coverage the interaction potential is a periodic repetition of the interaction potential in the $b$ range $[-1.585 \AA, 1.585 \AA]([-4.755 \AA, 1.585 \AA])$ for one adsorbate (Fig. 4). For $1 \mathrm{ML}$ coverage the attractive potential wells are fully occupied on these planes. As a consequence, the interaction potential is less attractive. We also analyze the $Y_{p}=X_{p}-1.585 \AA$ plane, which do not have any adsorbate filling a bridge adsorption site for $0.5 \mathrm{ML}$, while half of the bridge sites are covered for $1 \mathrm{ML}$ coverage. At $1.0 \mathrm{ML}$ coverage, the $Y_{p}=X_{p}-1.585 \AA$ 
plane and the parallel ones such as the $Y_{p}=X_{p}-4.755 \AA$ plane are equivalent. At $0.5 \mathrm{ML}$, half of the diagonal planes are equivalent to the shown $Y_{p}=X_{p}-1.585 \AA$ plane, but the other half are as the $Y_{p}=X_{p}-1.585 \AA$ plane at $1 \mathrm{ML}$. The negative values of the interaction potential are therefore reduced as the coverage increases and the projectiles will experience in average less attraction to the surface.

\subsection{Dynamics simulations details}

The normal incidence scattering of atomic hydrogen on H-preadsorbed W(100) surface is investigated within the zero coverage limit (single adsorbate) and at finite coverages of 0.5 and 1.0 ML. QCT calculations, which account for the adsorbates ZPE in the initial conditions, are run on the constructed CRP PES for an incidence energy of the projectile in the range of $0.1-5.0 \mathrm{eV}$. For finite coverages, a $6 \times 6$ cell with periodic boundary conditions is used in order to model a covered infinite surface. The classical equations of motion are integrated for one projectile and one adsorbate in the zero coverage limit and for one projectile and 18 and 36 adsorbates in the $\Theta=0.5$ and 1.0 ML coverages, respectively. The impinging $H$

atom (projectile) starts at $Z_{p}=7.0 \AA$, in the asymptotic region of the potential, while the initial positions and velocities of the adsorbates (targets) sample the configurational space associated to the ZPE as detailed in Refs. 73,74. For finite coverage calculations, the $\left(X_{p}, Y_{p}\right)$ initial position of the projectile is randomly sampled in the covered surface irreducible unit cell, i.e., $0<X_{p}<1.585 \AA$ and $0<Y_{p}<3.17 \AA$ for 0.5 ML coverage, and $0<X_{p}<1.585 \AA$ and $0<Y_{p}<1.585 \AA$ for 1 ML coverage. For the zero coverage limit, we use the same sampling area of Ref. 22. The possible exit channels of the simulations are defined as done in Refs. 84,85 for the zero coverage limit and as done in Ref. 26 for finite coverages. In the former simulations, only ER trajectories are analyzed. Recombination takes place whenever both atoms reach the initial height of the projectile with a positive diatom center-of-mass momentum along the surface normal, and with an interatomic distance $r<$ $2.2 \AA$. Among them, ER reaction occurs when the formed molecule moves definitively toward 

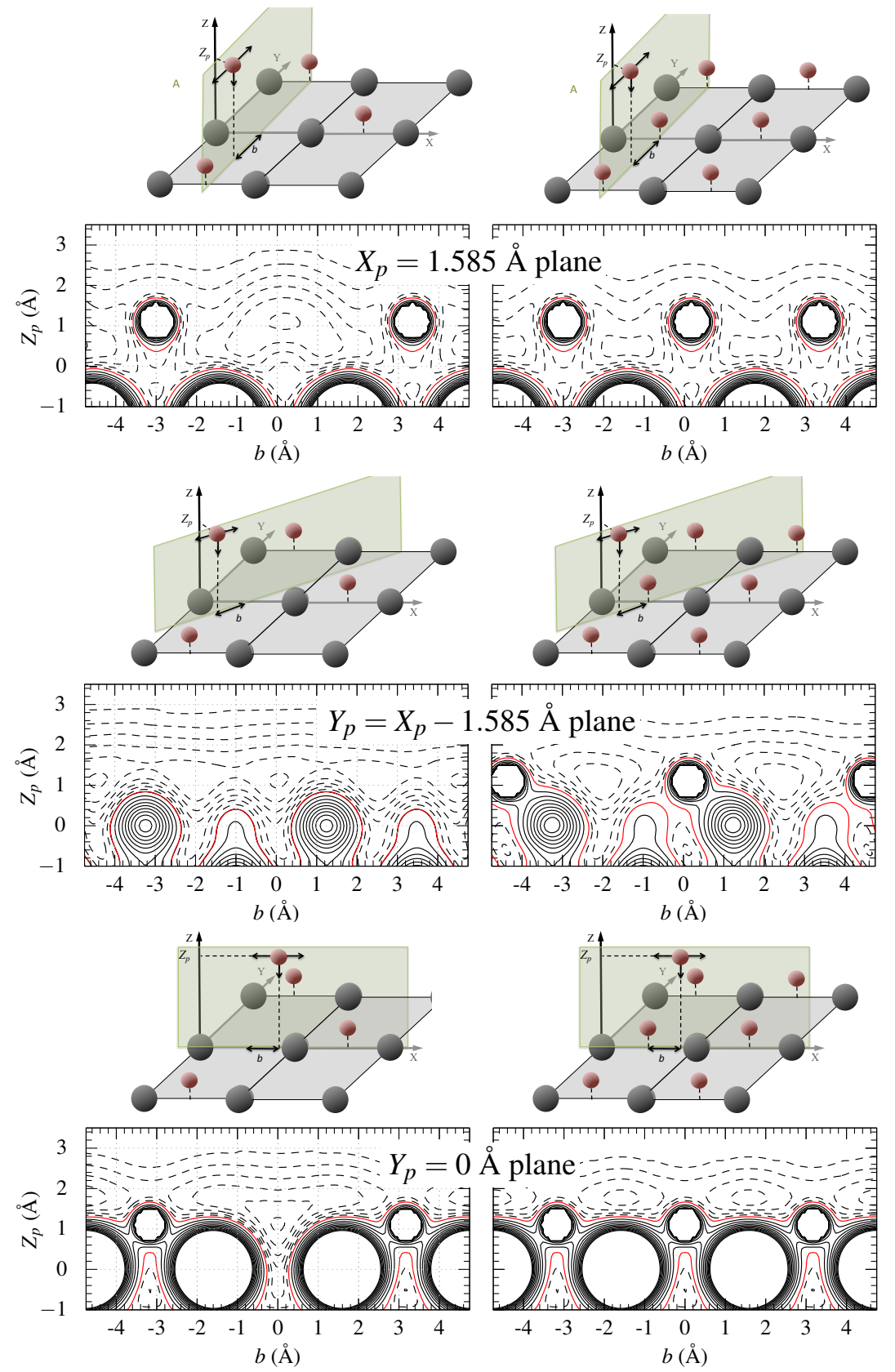

Figure 4: 2D $\left(b, Z_{p}\right)$-cuts of the multiadsorbate CRP PES for $\Theta=0.5 \mathrm{ML}$ (left) and 1.0 ML (right) coverages when the adsorbates sit in their equilibrium position and the projectile spans the indicated $\left(b, Z_{p}\right)$ plane. Full lines (dashed lines) are positive (negative) potential isovalues separated by $1 \mathrm{eV}(0.5 \mathrm{eV})$. Red lines correspond to the zero potential energy isovalue. The coordinate system and the and corresponding plane are depicted at the top of each 2D $\left(b, Z_{p}\right)$-cut. 
the vacuum before the second rebound of the projectile. In the finite coverage simulations, when an atom reaches the initial height of the projectile the event is defined as abstraction if the interatomic distance to any other atom is smaller than $2.2 \AA$ and as reflection otherwise. The rest of trajectories are integrated for 1 ps and classified as absorption if $Z_{p}<0$ and adsorption otherwise. It has been checked that a longer integration time does not change the results. Among the abstraction processes involving the projectile and one adsorbate, the primary HA differs from the above defined ER in that the projectile experiences more than one rebound before recombining with an adsorbate. When abstraction takes place involving two target atoms, it is classified as a secondary HA process. ${ }^{86}$

For the zero coverage limit, 368000 trajectories have been computed to ensure convergence, while 30000 (15000) trajectories have been computed for $\Theta=0.5$ ML (1.0 ML) coverage. As the multiadsorbate PES ignores any possible interaction involving three hydrogen atoms, trajectories are stopped whenever one $\mathrm{H}$ atom has two neighboring $\mathrm{H}$ atoms closer than $1.5 \AA$. The corresponding contribution is taken as an uncertainty to any possible outcome of scattering as done in Ref. 36 .

In order to rationalize the relevance of nonadiabatic effects upon scattering, molecular dynamics simulations are performed within the Born-Oppenheimer Static Surface approximation (BOSS) and the local-density friction approximation (LDFA). ${ }^{12,13,44}$ Whereas in the former energy exchange with the surface electrons is neglected, in the latter electronic nonadiabaticity is accounted for through a dissipative force in the classical equations of motion for the hydrogen atoms. To prevent leakage of the ZPE, the friction acts only when the energy of the preadsorbed atoms exceed the ZPE. On the ground that dissipation to electrons is largely dominating the relaxation of hydrogen on metals, ${ }^{24,25}$ dissipation to surface phonons is here neglected. 


\section{Results: the $\mathrm{H}-\mathrm{H}$ abstraction dynamics on $\mathrm{W}(100)$}

\subsection{Eley-Rideal dynamics in the zero coverage limit}

We start this section by comparing our BOSS QCT results in the zero coverage limit with those obtained from similar simulations performed on a FPLEPS PES. ${ }^{22,73}$ The objective is to evaluate the sensitivity of the ER dynamics to the PES representation. Thus, the ER cross sections $\sigma_{E R}$ as a function of the incidence energy of the projectile $\mathrm{E}_{i}$ for the FPLEPS and CRP PESs are shown in the top panel of Fig. 5. Although some differences between both PESs have been identified in section 2.1, these differences hardly affect the dependence of $\sigma_{E R}$ on $\mathrm{E}_{i}$. Sizable quantitative differences are only found at high $\mathrm{E}_{i}$. At these energies, the CRP PES predicts less reactivity than the FPLEPS PES.
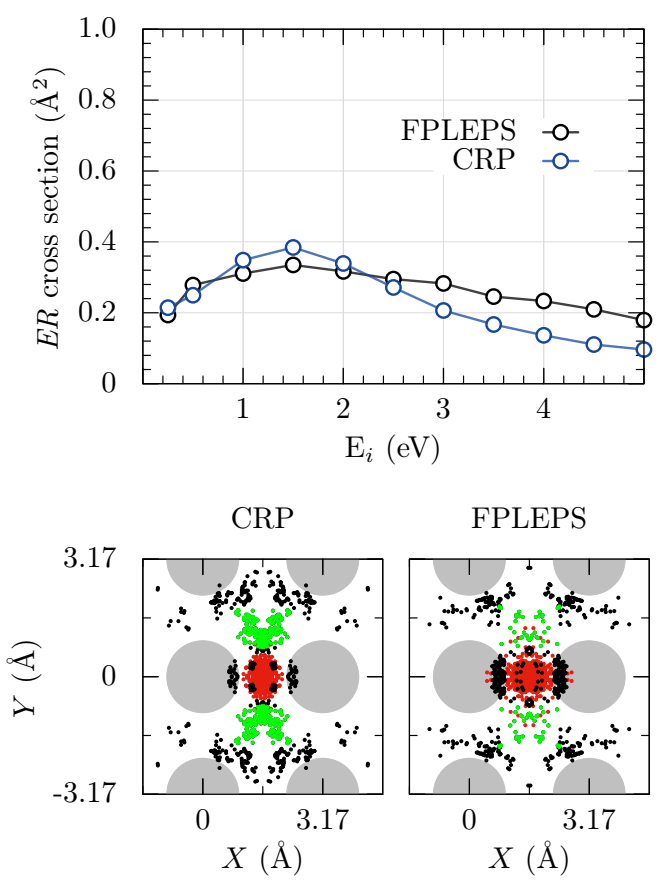

Figure 5: Top panel: BOSS cross sections for ER abstraction using the CRP (blue) and FPLEPS (black) PESs. Bottom panels: $\left(X_{p}, Y_{p}\right)$ turning positions of the projectiles leading to ER recombination at $\mathrm{E}_{i}=0.5 \mathrm{eV}$ for the CRP and FPLEPS PESs by rebounding on the first (black) and second (green) layer $\mathrm{W}$ atoms. The positions of the recombining target atoms are indicated by red points.

Even if ER reactivity is weakly affected by the representation of the PES at low $\mathrm{E}_{i}$, 
some dynamical changes are still observed in the ER abstraction process. As previously characterized in Ref. 74, ER reactive trajectories may proceed upon colliding with W atoms located in the first and second layers. The comparative analysis of the $\left(X_{p}, Y_{p}\right)$ turning points for the projectiles leading to $\mathrm{ER}$ recombination at $\mathrm{E}_{i}=0.5 \mathrm{eV}$ in both PESs (bottom panels of Fig. 5) shows the contribution of projectiles bouncing off the second layer W atoms (green points) at the expense of reaction through collision with the first layer W atoms (black points). For the CRP (FPLEPS) PES, the projectile goes below the target and catches it on its way back after colliding with a second layer W atom in 35\% (11\%) of ER trajectories. This is very likely due to the two differences identified in the static analysis of the PESs. On the one hand, ER reaction upon collision on the two closest $\mathrm{W}$ atoms to the target decreases due to the lower adsorption height of the adsorbate in the CRP PES $\left(Z_{t}=1.107 \AA\right.$ vs. $Z_{t}=1.2 \AA$ in the FPLEPS). On the other hand, projectiles are more efficiently scattered towards the target by the second layer $\mathrm{W}$ atoms due to the larger repulsive potential around them in the CRP PES (see Fig. 3). We note in passing that the latter dynamical difference is actually responsible of the lower absorption cross sections predicted by the CRP PES (not shown).

Fig. 6 displays the mean final translational $\left\langle\mathrm{E}_{\text {tran }}\right\rangle$, rotational $\left\langle\mathrm{E}_{\text {rot }}\right\rangle$, and vibrational energies $\left\langle\mathrm{E}_{v i b}\right\rangle$ of the ER-formed $\mathrm{H}_{2}$ molecules as a function of $\mathrm{E}_{i}$. Although there are some small quantitative discrepancies in the vibrational energy at high $\mathrm{E}_{i}$, the qualitative behavior is not affected by the PES representation.

\subsection{Abstraction dynamics on H-covered W(100)}

The probabilities obtained within the BOSS calculation for adsorption, reflection, absorption, and abstraction are displayed in the top panels of Fig. 7 as a function of $E_{i}$ for $\Theta=0.5$ and 1.0

ML coverages. Many similarities with the H dynamics on H-covered W(110 $)^{26,36}$ are found. The predominant outcome at low incidence $\mathrm{E}_{i}$, i.e., adsorption, highly decreases in favor of the absorption and reflection channels as $\mathrm{E}_{i}$ increases. As $\Theta$ increases, adsorption is favored 


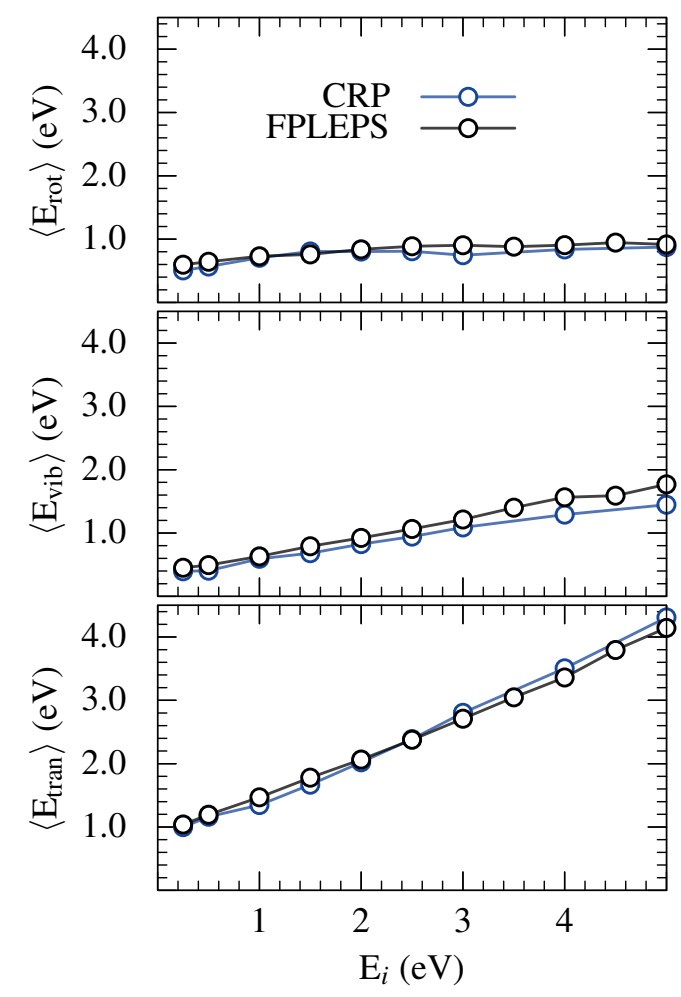

Figure 6: Final average translational $\left\langle\mathrm{E}_{\text {tran }}\right\rangle$, vibrational $\left\langle\mathrm{E}_{\text {vib }}\right\rangle$, and rotational energies $\left\langle\mathrm{E}_{\text {rot }}\right\rangle$ of the ER-formed $\mathrm{H}_{2}$ molecules as a function of $\mathrm{E}_{i}$. Results for FPLEPS (black) and CRP (blue) PESs are shown. 

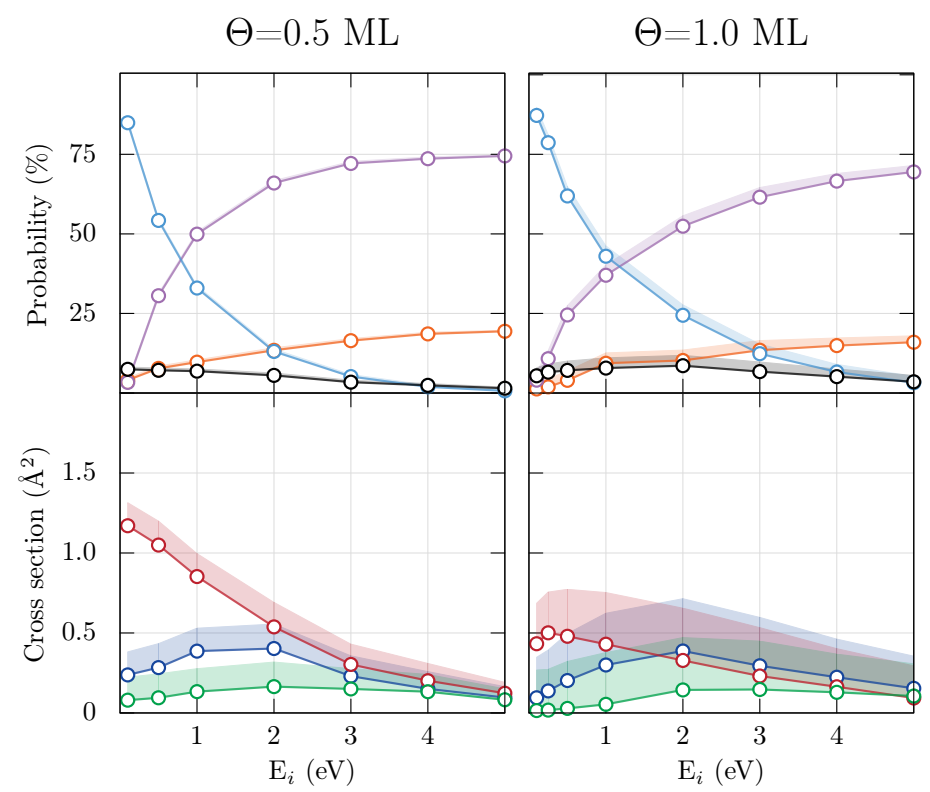

Figure 7: Top panels: probabilities for adsorption (blue), absorption (purple), reflection (orange) and abstraction (black) as a function of the projectile's incidence energy. Bottom panels: cross section for ER (blue), primary HA (red), and secondary HA (green) abstraction as a function of $\mathrm{E}_{i}$. Left and right panels correspond to 0.5 and 1.0 ML coverages, respectively. Uncertainties, which correspond to the contribution of stopped trajectories are represented by shaded domains.

due to the more efficient energy loss to adsorbates, whereas reflection of some projectiles is prevented due to it. Absorption probabilities decrease with coverage because the absorption path is through bridge sites, which are more populated.

Concerning the recombination mechanisms, the bottom panels of Fig. 7 display the cross sections per adsorbate for ER, primary HA and secondary HA abstraction as a function of $\mathrm{E}_{i}$. Within the BOSS approximation, HA mechanism dominates abstraction at low incidence energies and coverages, whereas as $\mathrm{E}_{i}$ and $\Theta$ increase the two mechanisms compete. Secondary HA contribution to the total cross section is small and decreases with coverage at low $\mathrm{E}_{i}$, while it is almost unchanged at high $\mathrm{E}_{i}$.

The comparison with the LDFA results in Fig. 8 helps to rationalize the effect of energy dissipation to the metal electrons. For the two coverages, accounting for $e-h$ pair excitations increases adsorption at the expense of reflection and abstraction (see top panels in Fig. 8). As in the W(110) surface, the reduction experienced by the abstraction cross section when 

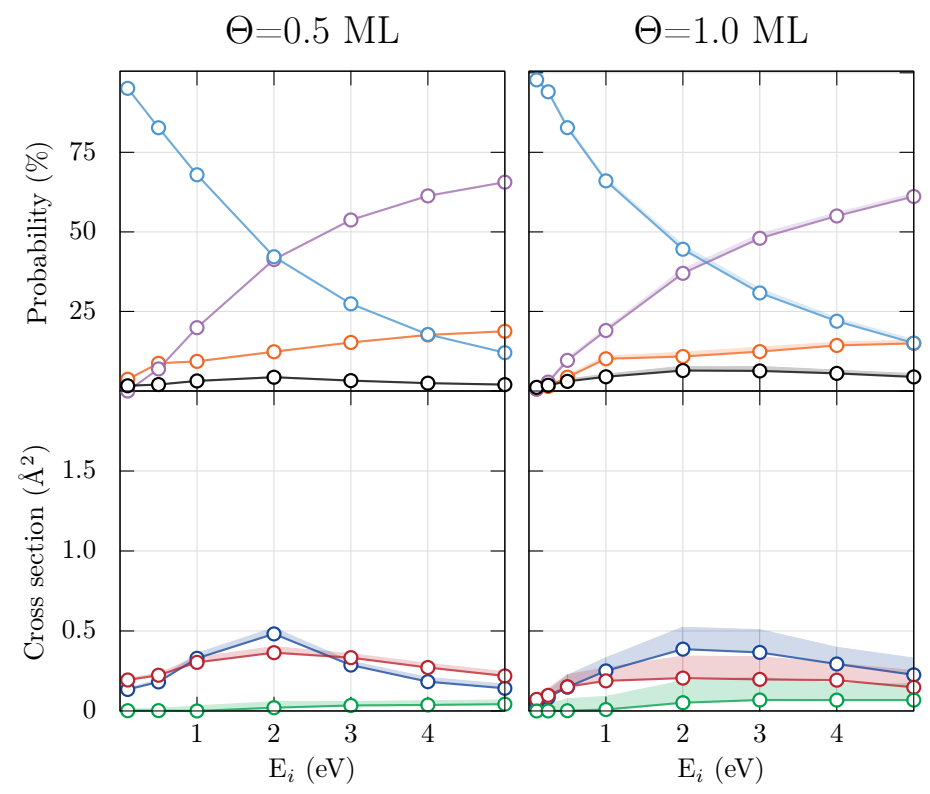

Figure 8: Same as in Fig. 7 but for LDFA calculation.

accounting for low energy electronic excitations is much more pronounced at the low coverage, and mainly originates from a reduction in the HA channels (see lower panels of Fig. 7 and 8). In this respect, we have verified that for low coverages the primary HA mechanism can take place via an initial collision with a second layer atom. This contribution amounts up to a $19 \%$ for $0.5 \mathrm{ML}$ coverage. Interestingly, inclusion of e-h pair excitations hardly affects this contribution. The already small contribution via secondary HA to abstraction decreases when including electronic excitations, particularly at $\Theta=0.5 \mathrm{ML}$ coverage, for which this contribution almost disappears. Overall, within the LDFA, abstraction cross sections slightly depend on coverage. At low coverage direct ER and HA mechanisms closely compete whatever the incidence energy of the projectile. As the coverage increases, ER becomes the dominant abstraction channel at $\mathrm{E}_{i}>1.0 \mathrm{eV}$.

The significant reduction of HA recombination caused by energy dissipation to $e-h$ pair excitations on W(100) stems, as well as in W(110), ${ }^{26}$ from the great reduction of the relaxation time for hot hydrogen atoms and concomitantly, the recombining hot-species lifetime. This is illustrated in Fig. 9 where the distribution of ER and primary HA abstraction times as obtained within the BOSS and LDFA simulations are displayed for $\Theta=0.5 \mathrm{ML}$ (left panels) 


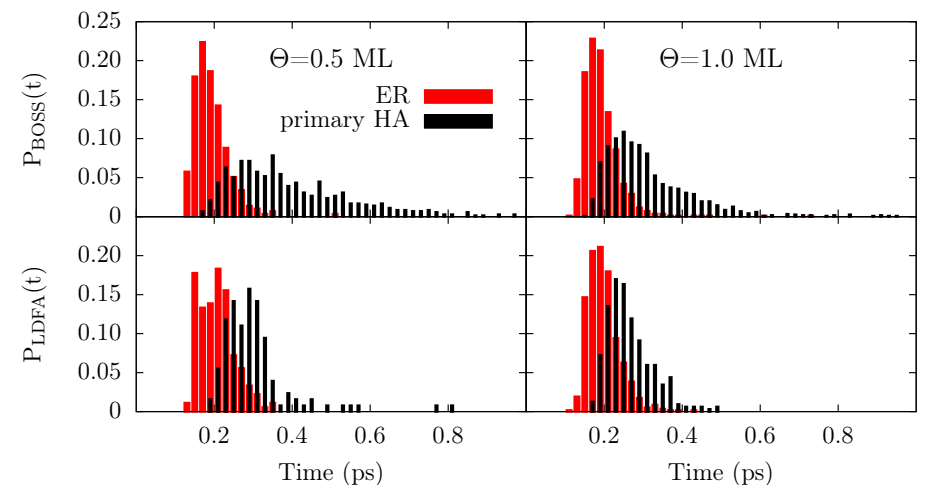

Figure 9: Normalized distribution of ER (red) and primary HA abstraction (black) times within the BOSS (top panels) and LDFA (bottom panels) simulations at $\Theta=0.5$ ML (left) and 1.0 ML coverages (right).

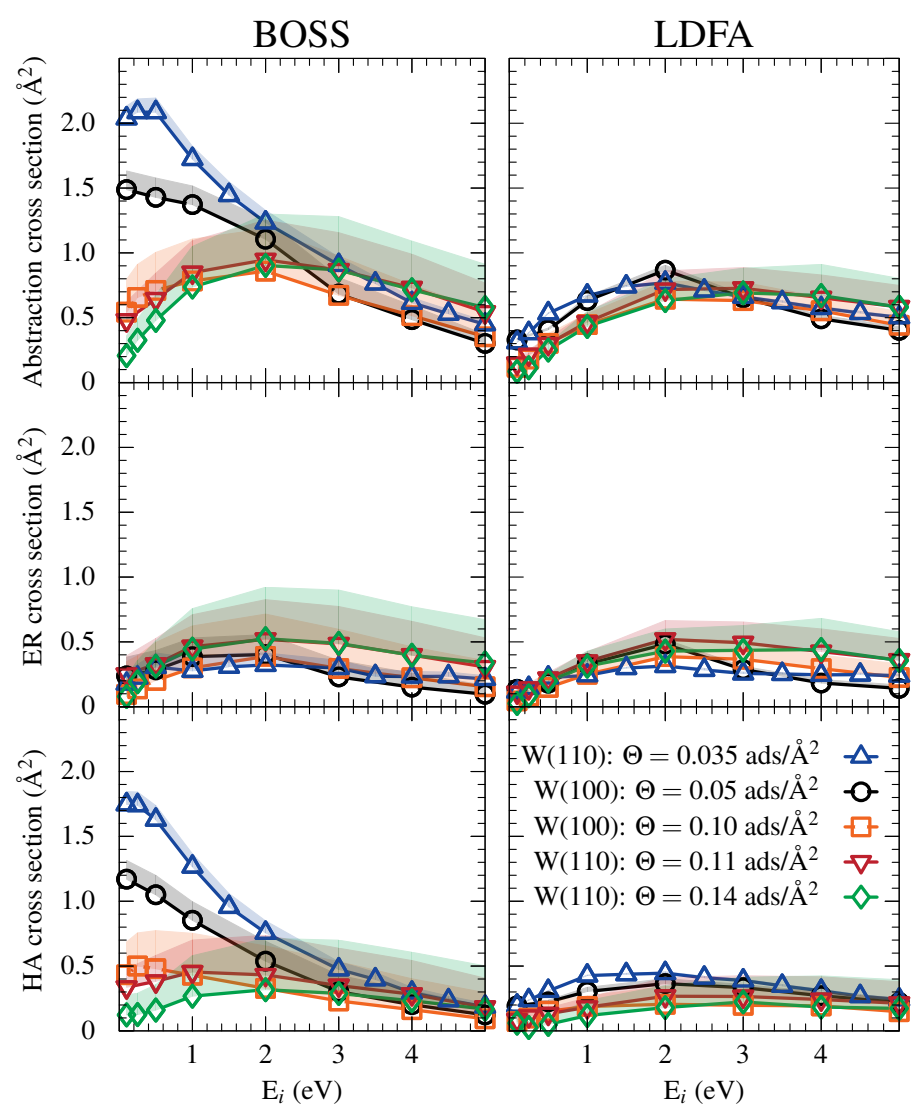

Figure 10: Cross sections for the total (top panels), ER (middle panels), and primary HA (bottom panels) abstraction processes as a function of $\mathrm{E}_{i}$ from BOSS (right) and LDFA (left) calculations. Different curves correspond to different coverages and W crystal surfaces, indicated in the key. Uncertainties, which correspond to the contribution of stopped trajectories (see text), are represented by shaded domains. 
and 1.0 ML (right panels) when $\mathrm{E}_{i}=0.5 \mathrm{eV}$. This time is taken as the total time for abstraction. At $\Theta=0.5 \mathrm{ML}$ coverage, when accounting for $e-h$ pair excitations, the timescale for both abstraction processes become similar. Concomitantly, the distances travelled on the surface before recombination become similar. At $\Theta=1.0 \mathrm{ML}$, the difference between the HA and ER reaction timescales in the BOSS simulations (right panels in Fig. 9) is not as large as for the $\Theta=0.5 \mathrm{ML}$. The reason for this is twofold. On the one hand, the projectile energy is more efficiently dissipated into the other adsorbates, resulting in a short lifetime for the hot species even when electronic excitations are not accounted for. On the other hand, the repulsive interaction between the projectile and adsorbates hinders the approach of the former. As a consequence, reactivity is less influenced by the coupling to $e-h$ pair excitations at high coverage, as illustrated by Fig. 7 and 8 .

In the following we analyze the dependence of the different recombination cross sections on the crystal face by comparing our results for W(100) and W(110). Concerning the ER cross sections, they do depend only very slightly on the crystal face and the coverage both with BOSS and LDFA models (see middle panels of Fig. 10). Regarding the HA abstraction channel, within the BOSS model, we observe a strong dependence on coverage at low $\mathrm{E}_{i}$ in both crystal faces. However, as it is demonstrated by the analysis performed in Fig. 10, this dependence is governed by the adsorbate areal density and is basically independent of the crystal face. Nevertheless, within the LDFA the dependence of the HA cross section on the coverage is strongly reduced. The reason is that the reduction of the HA cross section produced by $\mathrm{e}-\mathrm{h}$ pair excitations at low $\mathrm{E}_{i}$ is less effective when increasing the coverage. All in all, within LDFA, abstraction cross sections weakly depend on coverage and on the crystal face. In both systems, for low energies $\left(E_{i}<2.0 \mathrm{eV}\right)$ the abstraction cross sections per adsorbate decreases with coverage, whereas at higher energies reactivity is independent of the coverage. As a final remark, it is worth to mention that the low sensitivity to the crystal face when using the CRP PES is not due to the effect of electronic excitations, since it is also observed in the BOSS simulations. The somewhat larger ER cross sections found in the zero 
coverage limit for the $\mathrm{W}(100)$ surface $^{22,74}$ are a consequence of the $\mathrm{H}+\mathrm{H} / \mathrm{W}(110)$ PFLEPS PES errors. The latter causes an overestimation of about a factor two in the ER cross sections on this surface, ${ }^{73}$ while much smaller differences are found between the PFLEPS and CRP PESs calculations in the case of ER recombination on W(100) (see Sec. 3.1).

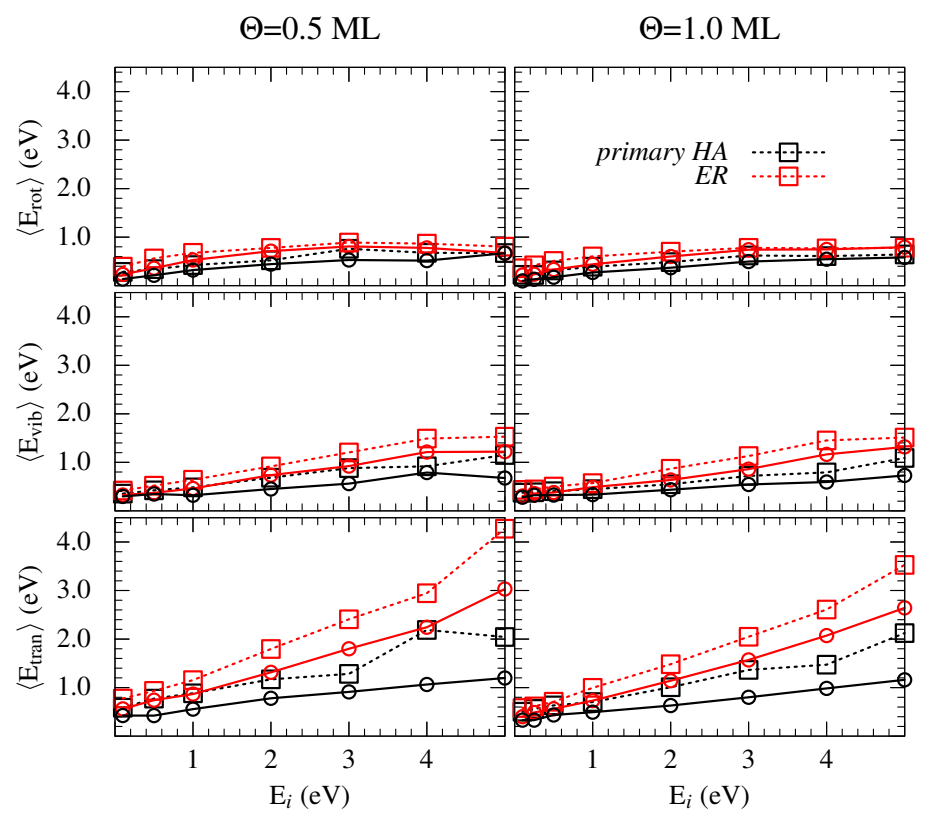

Figure 11: Final average translational $\left\langle\mathrm{E}_{\text {tran }}\right\rangle$, vibrational $\left\langle\mathrm{E}_{v i b}\right\rangle$, and rotational energies $\left\langle\mathrm{E}_{\text {rot }}\right\rangle$ of the ER (red) and HA (black) formed molecules as a function of the initial collision energy $\mathrm{E}_{i}$. Results from BOSS (squares) and LDFA (circles) simulations are shown.

Finally, we analyze the average energy distribution of the formed molecules. The final average translational $\left\langle\mathrm{E}_{\text {tran }}\right\rangle$, vibrational $\left\langle\mathrm{E}_{v i b}\right\rangle$, and rotational energies $\left\langle\mathrm{E}_{\text {rot }}\right\rangle$ of the ERformed and HA-formed $\mathrm{H}_{2}$ molecules are plotted in Fig. 11, as a function of the initial collision energy $\mathrm{E}_{i}$ at $\Theta=0.5$ (right panels) and 1.0 ML (right panels) coverages. Some general results are obtained that are independent of the coverage and also of the model used (BOSS or LDFA). First, as it could be expected, the final energy increases with $\mathrm{E}_{i}$ and ER-formed molecules carry always higher translational, vibrational and rotational energy than HA-formed molecules. We also observe that the increase of the $\left\langle\mathrm{E}_{\operatorname{tran}}\right\rangle$ with $\mathrm{E}_{i}$ is much more pronounced than those of $\left\langle\mathrm{E}_{v i b}\right\rangle$ and $\left\langle\mathrm{E}_{\text {rot }}\right\rangle$. Concerning the dependence on coverage of these, within the BOSS approximation $\left\langle\mathrm{E}_{v i b}\right\rangle$ and $\left\langle\mathrm{E}_{\text {rot }}\right\rangle$ hardly depend on $\Theta$, whereas 
$\left\langle\mathrm{E}_{\text {tran }}\right\rangle$ decreases with $\Theta$. Moreover, we observe that this decrease of $\left\langle\mathrm{E}_{\text {tran }}\right\rangle$ with $\Theta$ is very similar for both ER and HA abstraction. However, within LDFA $\left\langle\mathrm{E}_{\text {tran }}\right\rangle$ is strongly reduced. This reduction is more important at low $\Theta$. This makes also $\left\langle\mathrm{E}_{\text {tran }}\right\rangle$ independent of the coverage. As a consequence of all this, when $e-h$ pair excitations are considered, the final energies of ER- and HA-formed $\mathrm{H}_{2}$ molecules on W(100) are also insensitive to the coverage. This final statement can be generalized for the two different crystal faces. In Fig. 12 we show the average energy distributions for $\mathrm{H}_{2}$ molecules abstracted from W(100) and W(110) as a function of $\mathrm{E}_{i}$ for all studied coverages. This figure demonstrates that the partition of internal energy of $\mathrm{H}_{2}$ molecules abstracted from $\mathrm{W}(100)$ and $\mathrm{W}(110)$ depends on $\mathrm{E}_{i}$, but hardly does on the coverage and crystal face within the initial conditions studied in the present work.

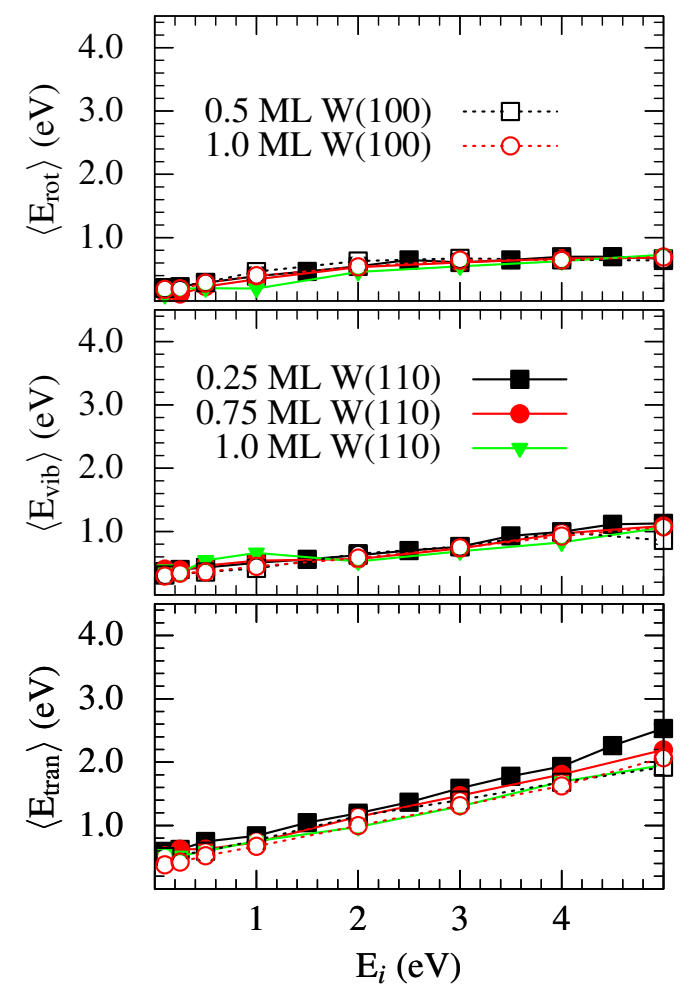

Figure 12: Final average translational $\left\langle\mathrm{E}_{\text {tran }}\right\rangle$, vibrational $\left\langle\mathrm{E}_{v i b}\right\rangle$, and rotational energies $\left\langle\mathrm{E}_{\text {rot }}\right\rangle$ of the abstracted $\mathrm{H}_{2}$ molecules formed in $\mathrm{W}(100)$ and $\mathrm{W}(110)$ as a function of $\mathrm{E}_{i}$ within LDFA. 


\section{Conclusions}

We have theoretically investigated hydrogen abstraction on a W(100) surface at finite coverages $(\Theta=0.5$ and $1.0 \mathrm{ML})$. In order to rationalize the effect of electron-hole (e-h) pair excitations, molecular dynamics simulations are performed within the Born-Oppenheimer Static Surface approximation (BOSS) and the local-density friction approximation (LDFA). Within the BOSS approximation, the Hot-Atom (HA) process dominates abstraction of $\mathrm{H}_{2}$ at low coverage $(\Theta=0.5 \mathrm{ML})$ and low collision energy $\left(\mathrm{E}_{i}<2.0 \mathrm{eV}\right)$. With increasing surface coverage $(\Theta=1.0 \mathrm{ML})$, HA versus Eley-Rideal (ER) balance changes to make ER the dominant mechanism. Moreover, the total abstraction time decreases and both primary HA and ER processes take place in a similar timescale at high coverages. ER and HA recombination processes produce vibrationally and rotationally hot molecules, however ER leads to the more excited ones. The average energy partition between rotation and vibration is weakly affected by coverage changes. Conversely, the mean final translational energies decrease when surface coverage increases because of scattering off other adsorbates before recombination.

The effect of $\mathrm{e}-\mathrm{h}$ pair excitations is to enhance adsorption at the expense of absorption, reflection and abstraction, but the qualitative evolution of the three former channels with $\mathrm{E}_{i}$ hardly changes. In contrast, abstraction is drastically reduced at low $\mathrm{E}_{i}$ and low coverage. The HA recombination mechanism, which is supposed to dominate recombination at low coverage and low $\mathrm{E}_{i}$, is shown to be significantly affected by the electronic excitations, since they greatly reduce the relaxation time and travelled length of hot hydrogen on the W(100) surface. As a result, the HA mechanism is considerably diminished in favor of $\mathrm{H}$ adsorption for these incidence conditions. Thus, the evolution of the abstraction channel with $\mathrm{E}_{i}$ changes at $\Theta=0.5 \mathrm{ML}$ coverage. As a matter of fact, within LDFA both ER and

HA mechanisms compete whatever the coverage, but as the coverage increases ER becomes the dominant abstraction channel. Within LDFA, the average energy partition between translation, rotation, and vibration is weakly affected by coverage changes.

The comparison of LDFA calculations of $\mathrm{H}$ abstraction from W(100) and W(110) surfaces 
shows low sensitivity on the crystal face, both the reactivity and final energy distributions of the formed molecules are similar. Moreover, within LDFA, ER and primary HA dynamics share many similarities at all initial conditions studied here. Both abstraction processes take place on the same timescale and the travelled length by the projectiles before recombination are similar, thus highlighting the arbitrary character of their separation. Primary recombination on $\mathrm{W}(100)$ and $\mathrm{W}(110)$ might be considered as a unique reactive process.

\section{Conflicts of interest}

There are no conflicts of interest to declare

\section{Acknowledgements}

O.G., J.I.J, and M.A. acknowledge financial support by the Basque Departamento de Educación, Universidades e Investigación, the University of the Basque Country UPV/EHU (Grant No IT-756-13) and the Spanish Ministerio de Economía y Competitividad (Grant No. FIS2016-76471-P). O.G., M.A., and P.L. acknowledge the IDEX Bordeaux (ANR-10IDEX-03-02) and Euskampus for fundings. Computational resources were provided by the DIPC computing center and the Mésocentre de Calcul Intensif Aquitain (MCIA). Research has been conducted in the scope of the Transnational Common Laboratory "QuantumChemPhys: Theoretical Chemistry and Physics at the Quantum Scale".

\section{References}

(1) J. K. Nørskov, T. Bligaard, J. Rossmeisl and C. H. Christensen, Nat. Chem., 2009, 1, 37-46.

(2) C. Stampfl, M. V. Ganduglia-Pirovano, K. Reuter and M. Scheffler, Surf. Sci., 2002, 500, 368-394. 
(3) A. Groß, Surf. Sci. Rep., 1998, 32, $291-340$.

(4) P. Nieto, E. Pijper, D. Barredo, G. Laurent, R. A. Olsen, E.-J. Baerends, G.-J. Kroes and D. Farías, Science, 2006, 312, 86-89.

(5) C. Díaz, E. Pijper, R. A. Olsen, H. F. Busnengo, D. J. Auerbach and G. J. Kroes, Science, 2009, 326, 832-834.

(6) P. M. Hundt, B. Jiang, M. E. van Reijzen, H. Guo and R. D. Beck, Science, 2014, 344, 504-507.

(7) X. J. Shen, A. Lozano, W. Dong, H. F. Busnengo and X. H. Yan, Phys. Rev. Lett., 2014, 112, 046101.

(8) I. Goikoetxea, J. Meyer, J. I. Juaristi, M. Alducin and K. Reuter, Phys. Rev. Lett., 2014, 112, 156101.

(9) E. N. Ghassemi, M. Wijzenbroek, M. F. Somers and G.-J. Kroes, Chem. Phys. Lett., 2017, 329-335.

(10) H. Nienhaus, Surf. Sci. Rep., 2002, 45, 1.

(11) A. M. Wodtke, Chem. Soc. Rev., 2016, 45, 3641-3657.

(12) M. Alducin, R. Díez Muiño and J. I. Juaristi, Progr. Surf. Sci., 2017, 92, 317 - 340.

(13) J. I. Juaristi, M. Alducin, R. Díez Muiño, H. F. Busnengo and A. Salin, Phys. Rev. Lett., 2008, 100, 116102.

(14) I. Goikoetxea, J. I. Juaristi, M. Alducin and R. Díez Muiño, J. Phys.: Condens. Matter, 2009, 21, 264007.

(15) G. Füchsel, M. del Cueto, C. Díaz and G.-J. Kroes, J. Phys. Chem. C, 2016, 120, $25760-25779$. 
(16) B. Jiang, M. Alducin and H. Guo, J. Phys. Chem. Lett., 2016, 7, 327-331.

(17) X. Luo, B. Jiang, J. I. Juaristi, M. Alducin and H. Guo, J. Chem. Phys., 2016, 145, 044704 .

(18) R. J. Maurer, B. Jiang, H. Guo and J. C. Tully, Phys. Rev. Lett., 2017, 118, 256001.

(19) I. Lončarić, G. Füchsel, J. I. Juaristi and P. Saalfrank, Phys. Rev. Lett., 2017, 119, 146101.

(20) M. Pavanello, D. J. Auerbach, A. M. Wodtke, M. Blanco-Rey, M. Alducin and G.-J. Kroes, J. Phys. Chem. Lett., 2013, 4, 3735-3740.

(21) O. Bünermann, H. Jiang, Y. Dorenkamp, A. Kandratsenka, S. M. Janke, D. J. Auerbach and A. M. Wodtke, Science, 2015, 350, 1346-1349.

(22) O. Galparsoro, R. Pétuya, J. I. Juaristi, C. Crespos, M. Alducin and P. Larrégaray, J. Phys. Chem. C, 2015, 119, 15434-15442.

(23) G.-J. Kroes, J. I. Juaristi and M. Alducin, J. Phys. Chem. C, 2017, 121, 13617-13633.

(24) M. Blanco-Rey, J. I. Juaristi, R. Díez Muiño, H. F. Busnengo, G. J. Kroes and M. Alducin, Phys. Rev. Lett., 2014, 112, 103203.

(25) D. Novko, M. Blanco-Rey, J. I. Juaristi and M. Alducin, Phys. Rev. B, 2015, 92, 201411.

(26) O. Galparsoro, R. Pétuya, F. Busnengo, J. I. Juaristi, C. Crespos, M. Alducin and P. Larrégaray, Phys. Chem. Chem. Phys., 2016, 18, 31378.

(27) O. Galparsoro, H. F. Busnengo, J. I. Juaristi, C. Crespos, M. Alducin and P. Larregaray, J. Chem. Phys., 2017, 147, 121103.

(28) D. Novko, I. Lončarić, M. Blanco-Rey, J. I. n. Juaristi and M. Alducin, Phys. Rev. B, 2017, 96, 085437. 
(29) D. Kolovos-Vellianitis and J. Küppers, Surf. Sci., 2004, 548, 67-74.

(30) B. Jackson, X. Sha and Z. B. Guvenc, J. Chem. Phys., 2002, 116, 2599-2608.

(31) T. Kammler, J. Lee and J. Küppers, J. Chem. Phys., 1997, 106, 7362-7371.

(32) J. Y. Kim and J. Lee, J. Chem. Phys., 2000, 113, 2856-2865.

(33) D. V. Shalashilin and B. Jackson, J. Chem. Phys., 1998, 109, 2856-2864.

(34) Z. B. Guvenc, X. Sha and B. Jackson, J. Chem. Phys., 2001, 115, 9018-9027.

(35) D. V. Shalashilin, B. Jackson and M. Persson, J. Chem. Phys., 1999, 110, 11038-11046.

(36) R. Pétuya, P. Larrégaray, C. Crespos, P. Aurel, H. F. Busnengo and A. E. Martínez, J. Phys. Chem. C, 2015, 119, 3171-3179.

(37) M. Head-Gordon and J. C. Tully, J. Chem. Phys., 1995, 103, 10137-10145.

(38) J. R. Trail, D. M. Bird, M. Persson and S. Holloway, J. Chem. Phys., 2003, 119, $4539-4549$.

(39) M. Grotemeyer and E. Pehlke, Phys. Rev. Lett., 2014, 112, 043201.

(40) M. Askerka, R. J. Maurer, V. S. Batista and J. C. Tully, Phys. Rev. Lett., 2016, 116, 217601.

(41) D. Novko, M. Alducin, M. Blanco-Rey and J. I. Juaristi, Phys. Rev. B., 2016, 94, 224306.

(42) S. P. Rittmeyer, J. Meyer and K. Reuter, Phys. Rev. Lett., 2017, 119, 176808.

(43) D. Novko, M. Alducin and J. I. Juaristi, Phys. Rev. Lett., 2018, 120, 156804.

(44) M. Alducin, R. Díez Muiño and J. I. Juaristi, in Non-Adiabatic Effects in Gas-Surface Dynamics: The Local Density Friction Approximation, ed. M. Rocca, T. Rahman and L. Vattuone, Springer, 2018. 
(45) L. Martin-Gondre, G. A. Bocan, M. Alducin, J. I. Juaristi and R. Díez Muiño, Comp. Theor. Chem., 2012, 990, 126-131.

(46) M. A. D. Novko, M. Blanco-Rey and J. I. Juaristi, Phys. Rev. B, 2016, 93, 245435.

(47) L. Martin-Gondre, M. Alducin, G. A. Bocan, R. Díez Muiño and J. I. Juaristi, Phys. Rev. Lett., 2012, 108, 096101.

(48) A. S. Muzas, J. I. Juaristi, M. Alducin, R. Díez Muiño, G. Kroes and C. Díaz, J. Chem. Phys., 2012, 137, 064707.

(49) L. Zhou, X. Zhou, M. Alducin, L. Zhang, B. Jiang and H. Guo, J. Chem. Phys., 2018, 148, 014702 .

(50) O. Galparsoro, J. I. Juaristi, C. Crespos, M. Alducin and P. Larrégaray, J. Phys. Chem. C, 2017, 121, 19849-19858.

(51) G. Füchsel, T. Klamroth, S. Monturet and P. Saalfrank, Phys. Chem. Chem. Phys., 2011, 13, 8659-8670.

(52) I. Lončarić, M. Alducin, P. Saalfrank and J. I. Juaristi, Phys. Rev. B, 2016, 93, 014301.

(53) R. Scholz, G. Floß, P. Saalfrank, G. Füchsel, I. Lončarić and J. I. Juaristi, Phys. Rev. B., 2016, 94, 165447.

(54) J. I. Juaristi, M. Alducin and P. Saalfrank, Phys. Rev. B, 2017, 95, 125439.

(55) H. F. Busnengo, A. Salin and W. Dong, J. Chem. Phys., 2000, 112, 7641-7651.

(56) G. Kresse, Phys. Rev. B, 2000, 62, 8295.

(57) R. A. Olsen, H. F. Busnengo, A. Salin, M. F. Somers, G. J. Kroes and E. J. Baerends, J. Chem. Phys., 2002, 116, 3841-3855.

(58) H. F. Busnengo and A. E. Martínez, J. Phys. Chem. C, 2008, 112, 5579-5588. 
(59) G. Kresse and J. Hafner, Phys. Rev. B, 1993, 47, 558.

(60) G. Kresse and J. Hafner, Phys. Rev. B, 1994, 49, 14251.

(61) G. Kresse and J. Hafner, J. Phys. Condens. Mat., 1994, 6, 8245.

(62) G. Kresse and J. Furthmüller, Comut. Mater. Sci., 1996, 6, 15-50.

(63) G. Kresse and J. Furthmüller, Phys. Rev. B, 1996, 54, 11169.

(64) J. P. Perdew, P. Ziesche and H. Eschrig, Electronic structure of solids, Akademie Verlag, Berlin, 1991, vol. 11.

(65) J. P. Perdew and Y. Wang, Phys. Rev. B, 1992, 46, 12947.

(66) D. Vanderbilt, Phys. Rev. B, 1990, 41, 7892.

(67) H. Froitzheim, H. Ibach and S. Lehwald, Phys. Rev. Lett., 1976, 36, 1549.

(68) U. Jayasooriya, M. Chesters, M. Howard, S. Kettle, D. Powell and N. Sheppard, Surf. Sci., 1980, 93, 526-534.

(69) H.-J. Herlt and E. Bauer, Surf. Sci., 1986, 175, 336-368.

(70) W. Ho, R. F. Willis and E. W. Plummer, Phys. Rev. Lett., 1978, 40, 1463-1466.

(71) W. Ho, R. Willis and E. Plummer, Phys. Rev. B, 1980, 21, 4202.

(72) P. Estrup and J. Anderson, J. Chem. Phys., 1966, 45, 2254-2260.

(73) R. Pétuya, P. Larrégaray, C. Crespos, H. F. Busnengo and A. E. Martínez, J. Chem. Phys., 2014, 141, 024701.

(74) R. Pétuya, C. Crespos, E. Quintas-Sánchez and P. Larrégaray, J. Phys. Chem. C, 2014, 118, 11704-11710.

(75) R. Biswas and D. Hamann, Phys. Rev. Lett., 1986, 56, 2291. 
(76) L. Lou, D. Langreth and P. Nordlander, Surf. Sci., 1990, 234, 412-420.

(77) J. Woods and J. Erskine, J. Vac. Sci. Technol. A, 1986, 4, 1414-1415.

(78) M. R. Barnes and R. F. Willis, Phys. Rev. Lett., 1978, 41, 1729-1733.

(79) L. Martin-Gondre, C. Crespos, P. Larrégaray, J. Rayez, B. van Ootegem and D. Conte, Chem. Phys. Lett., 2009, 471, $136-142$.

(80) L. Martin-Gondre, C. Crespos, P. Larrégaray, J. C. Rayez, B. van Ootegem and D. Conte, J. Chem. Phys., 2010, 132, 204501.

(81) L. Martin-Gondre, C. Crespos, P. Larrégaray, J. Rayez, D. Conte and B. van Ootegem, Chem. Phys., 2010, 367, 136-147.

(82) D. V. Shalashilin, B. Jackson and M. Persson, Faraday Discuss., 1998, 110, 287-300.

(83) Z. Piazza, M. Ajmalghan, Y. Ferro and R. Kolasinski, Acta Mater., 2018, 145, 388-398.

(84) E. Quintas-Sánchez, P. Larrégaray, C. Crespos, L. Martin-Gondre, J. Rubayo-Soneira and J. C. Rayez, J. Chem. Phys., 2012, 137, 064709.

(85) E. Quintas-Sánchez, C. Crespos, P. Larrégaray, J. C. Rayez, L. Martin-Gondre and J. Rubayo-Soneira, J. Chem. Phys., 2013, 138, 024706.

(86) R. Martinazzo, S. Assoni, G. Marinoni and G. F. Tantardini, J. Chem. Phys., 2004, 120, 8761-8771. 